\title{
The fatty acid translocase CD36 could be burden at life's winter
}

\author{
Friedrich Luft
}

Published online: 8 April 2011

(C) Springer-Verlag 2011

Cluster of differentiation 36 (CD36), also known as the thrombospondin receptor, fatty acid translocase, scavenger receptor class B member 3 (growth factor GP88), glycoprotein $\mathrm{IV}$, and glycoprotein IIIb, is an integral membrane protein present in many types of mammalian cells. CD36 binds numerous ligands including collagen, thrombospondin, damaged erythrocytes, oxidized low-density lipoprotein, phospholipids, and long-chain fatty acids. I first became aware of CD36 when I encountered genetic studies in rodents that implicated the CD36 gene locus in cardiovascular disease [1]. These studies showed that in spontaneously hypertensive rats (SHR), genetic variance on CD36 influenced ectopic fat and fat metabolite accumulation in the metabolic syndrome (waist fat, blood pressure, insulin resistance, triglyceridemia). The peroxisome proliferatoractivated receptor- $\gamma$ (PPAR- $\gamma$ ) pathway is a drug target that influences these phenotypes [1]. The studies also suggested that angiotensin II receptor blockers with the ability to selectively modulate PPAR- $\gamma$ activity could influence gene expression in these fat metabolism pathways and could represent useful prototypes for a new class of transcriptionmodulating drugs. Perhaps, such drugs could be aimed at treating patients with hypertension and metabolic syndrome. In their original study, Aitman et al. combined cDNA microarrays, congenic mapping, and radiation hybrid mapping, to identify a defective $C d 36$ gene in SHR. Thus, the rats that have no (or less) CD36 expression, apparently

F. Luft $(\bowtie)$

Experimental and Clinical Research Center, Charité Medical Faculty and Max-Delbrück Center for Molecular Medicine,

Robert-Rössle Strasse 10,

13125, Berlin, Germany

e-mail: luft@charite.de exhibit increased blood pressure, cardiac hypertrophy, and all aspects of the metabolic syndrome [2].

The CD36 protein belongs to the class B scavenger receptor family that includes receptors for selective cholesteryl ester uptake, scavenger receptor class B type I, and lysosomal integral membrane protein II [3]. CD36 may be particularly important for macrophages. On macrophages, CD36 forms part of a non-opsonic receptor (the scavenger receptor CD36/alphaV beta3 complex) and is involved in phagocytosis [4]. CD36 is directly implicated in numerous pathophysiological processes, including hemostasis, thrombosis, propensity to acquire malaria, inflammation, lipid metabolism, and atherogenesis. A schematic view of CD36 with its two transmembrane domains is shown (Fig. 1). When CD36 binds a ligand, the protein and ligand are internalized. This internalization is independent of macropinocytosis and occurs by an actin-dependent mechanism requiring the activation of Src family kinases, JNK, and Rhofamily GTPases. The process is not affected by phosphatidylinositol 3-kinase inhibitors and does not involve $\mathrm{Na}^{+} / \mathrm{H}^{+}$ exchange. The CD36 amino acid sequence predicts a hydrophobic region near each end of the protein large enough to span cellular membranes. CD36 is predicted to have a "hairpin-like" structure with $\alpha$-helices at the $\mathrm{C}$ - and $\mathrm{N}$-termini, projecting through the membrane and a larger extracellular loop (Fig. 1). This topology prediction is supported by transfection experiments in cultured cells using deletion mutants of CD36. Disulfide linkages between four of the six cysteine residues in the extracellular loop are required for efficient intracellular processing and transport of CD36 to the plasma membrane. However, what role these linkages play on the function of the mature $\mathrm{CD} 36$ protein on the cell surface is not clear. CD36 may be regulated by posttranslational modifications. For instance, the protein is outfitted with four palmitoyl chains, two on each of the 
Fig. 1 Shown is the predicted topology of CD36, also known the thrombospondin receptor and other aliases, with two transmembrane-spanning domains. G, P, and A are glycosylation, phosphorylation, and acetylation (palmitoyl chains) sites, respectively, of this highly regulated protein (see reference 8 for details). Brackets refer to the presence of disulfide linkages

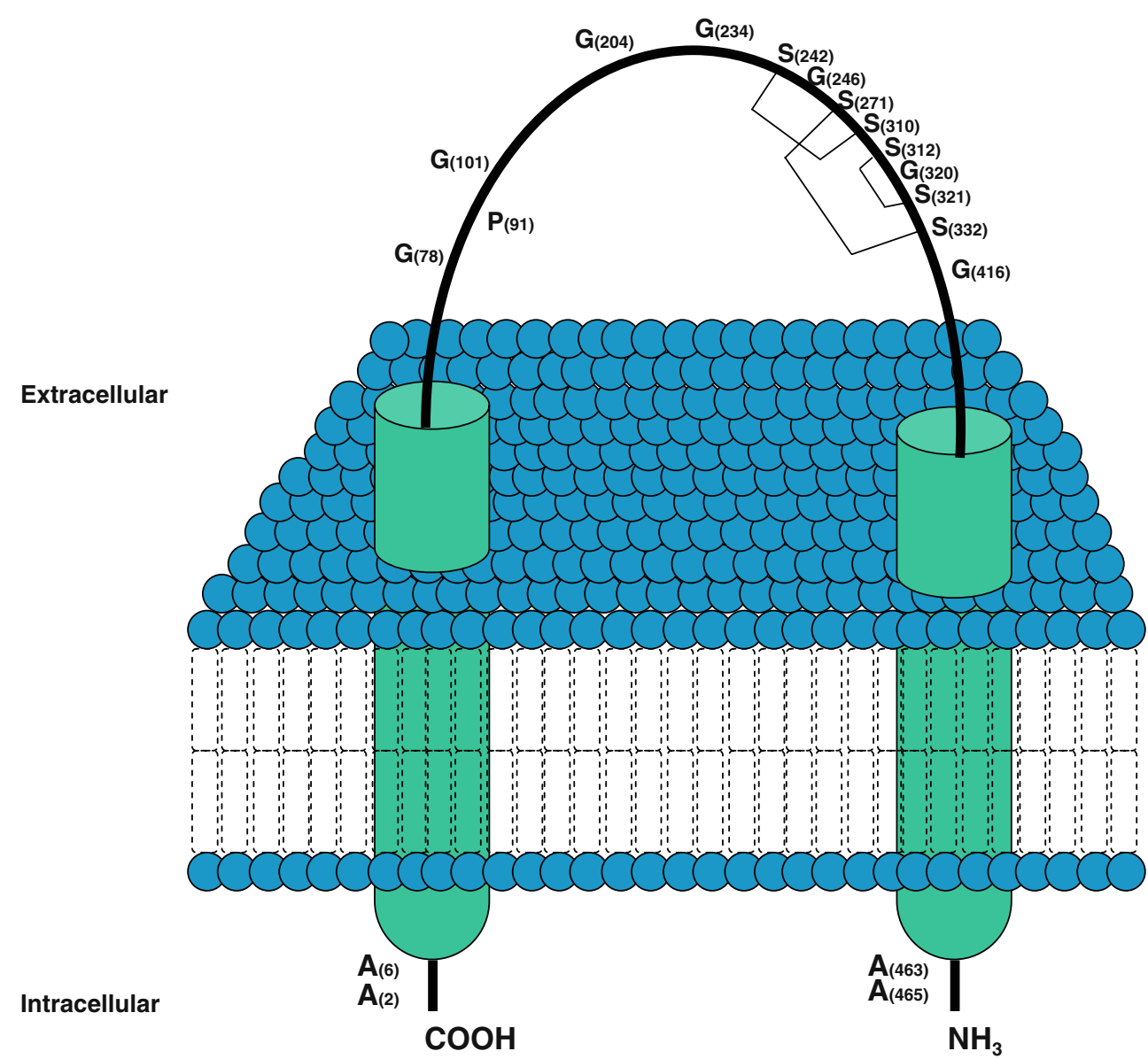

two intracellular domains. The function of these lipid modifications is currently unknown, but they may likely promote the association of CD36 with the membrane and possibly lipid rafts, which appear to be important for some CD36 functions. Recent work using genetically modified rodents has identified a clear role for CD36 in fatty acid and glucose metabolism, heart disease, taste, and dietary fat processing in the intestine. Thus, CD36 may be involved in glucose intolerance, atherosclerosis, arterial hypertension, diabetes, cardiomyopathy, and Alzheimer's disease, all in all, an impressive list of chronic conditions [5].

In this issue, Sung et al. [6] examined the age-related functional significance of CD36. They studied mice aged 10-12 weeks (young adults or "young" mice) and mice aged 40-44 weeks (middle-aged or "old" mice) and fed these mice either a low-fat or a high-fat diet for 12 weeks. Old mice developed more cardiac hypertrophy than young mice in response to a high-fat diet, albeit without cardiac malfunction. They also exhibited increased CD36 expression in their hearts with high-fat diet, which young mice did not. Interestingly, CD36 knockout mice were protected from high-fat diet-induced cardiac hypertrophy. The authors next directed their attention to issues of cardiac growth. They found that ceramide levels were increased in hearts of old mice given high-fat diet, compared to controls, while the adenosine monophosphate-induced protein kinase (AMPK) was inhibited under these conditions. The decreased AMPK activity was associated with the activation of mammaliantarget of rapamycin (mTOR) pathway. The mTOR-signaling pathway is responsible for proliferation, growth, and antiapoptosis. The notion here is that increased CD36 activity in old mice permits enhanced ceramide entry into cardiac myocytes that downregulates AMPK, which allows mTOR to function in an uninhibited fashion, promoting cardiac hypertrophy. In support of this idea, the authors present evidence that the S6 kinase (S6K1) was activated in old CD36 wild-type mice given high-fat diet, compared to old low-fat diet mice. Nonetheless, the eEF2 transcription factor was not changed, in terms of activity. The authors speculate that S6K1 is responsible for the effects they observed. The implications of the authors' findings are important for aging hearts. In such individuals, CD36 may be upregulated (increased in activity) in response to the "unhealthy" highfat diet. This behavior could evoke changes in AMPK phosphorylation and could in turn upregulate mTOR in the heart, which might contribute to cardiac hypertrophy, an important risk factor for cardiovascular events and mortality. 
How do these findings influence our thinking about CD36 and cardiovascular risk, aging, high-fat diets, etc.? No doubt, CD36 is strategically located. The molecule belongs to the "pattern recognition receptors" associated with innate immunity. CD36 sits on many cells, including macrophages [7]. CD36, toll-like receptor-4, natural antibodies, and $\mathrm{C}$-reactive protein all recognize common oxidation-specific pathogen-associated molecular patterns or "damage-associated molecular patterns", such as oxidized phospholipids and oxidized cholesteryl esters, and mediate a variety of immune responses. The responses range from expression of proinflammatory genes to excessive intracellular lipoprotein accumulation and to atheroprotective humoral immunity. The question is raised, "Why would high-fat diet increase CD36 expression and result in adverse signaling?" As I mentioned in the beginning, CD36 showed up in a genetic screen of SHR and Brown-Norway crosses, coupled with expression profiling. In those studies, the gene was highly expressed in adipose tissue of the SHR chromosome 4 congenic strain, but showed little or no expression in adipose tissue of the SHR progenitor (sick) strain. The defect here was related to the reduced expression of $\mathrm{CD} 36$, rather than to the increased expression of the protein [2]. These studies were not cited or discussed in the report by Sung et al. [6].

Sung et al. [6] provide evidence that the increased CD36 signaling in their model resulted in increased mTOR activity via reduction in AMPK. The findings would allow several interesting therapeutic controls for their hypothesis. Of interest would be if the AMPK stimulator, metformin reverses the state of affairs that the authors describe. Also, important to know would be whether or not sirolimus or other mTOR inhibitors would be therapeutically effective. Retinoids would be additional candidates. Also, unanswered by these experiments is the question of how highfat diet increases CD36 expression. The issue is particularly germane since high-fat weight-loss (Atkins) diets are now extremely popular. Does the authors' high-fat diet perform its effects independent of weight loss?

CD36 is a PPAR- $\gamma$ target gene. Pravenec and Kurtz had speculated that a PPAR- $\gamma$ activity might improve a lot of their cardiovascular disease model, the SHR [1]. Interestingly, a small ubiquitin modulator (SUMO) modification selectively regulates transcriptional activity of PPAR- $\gamma$. Chung et al. recently found that a SUMO-specific protease effectively de-SUMOylates PPAR $\gamma$-SUMO conjugates [7].
Overexpression of this protease in cell culture increased the expression of PPAR- $\gamma$ target genes, including CD36, both in the absence and presence of rosiglitazone. Thus, PPAR- $\gamma$ expression in response to high-fat diet could have been examined. The regulation of the $C D 36$ gene itself is also not trivial. The CD36 gene is located on chromosome 7 q11.2 and is encoded by 15 exons. Rać et al. (Fig. 1) summarized current knowledge of the CD36 gene structure, splicing, known mutations, and the molecular, metabolic, and clinical consequences of $C D 36$ gene variation [8]. In any event, there is much work to be done here and the current report leaves many questions unanswered in this burgeoning field.

Respectfully,

Friedrich C. Luft

\section{References}

1. Pravenec M, Kurtz TW (2007) Molecular genetics of experimental hypertension and the metabolic syndrome: from gene pathways to new therapies. Hypertension 49:941-952

2. Aitman TJ, Glazier AM, Wallace CA, Cooper LD, Norsworthy PJ, Wahid FN, Al-Majali KM, Trembling PM, Mann CJ, Shoulders CC, Graf D, St Lezin E, Kurtz TW, Kren V, Pravenec M, Ibrahimi A, Abumrad NA, Stanton LW, Scott J (1999) Identification of $\mathrm{Cd} 36$ (Fat) as an insulin-resistance gene causing defective fatty acid and glucose metabolism in hypertensive rats. Nat Genet 21:76-83

3. Schwenk RW, Holloway GP, Luiken JJ, Bonen A, Glatz JF (2010) Fatty acid transport across the cell membrane: regulation by fatty acid transporters. Prostaglandins Leukot Essent Fatty Acids 82:149-154

4. Silverstein RL, Febbraio M (2009) CD36, a scavenger receptor involved in immunity, metabolism, angiogenesis, and behavior. Sci Signal 2(72):re3

5. Areschoug T, Gordon S (2009) Scavenger receptors: role in innate immunity and microbial pathogenesis. Cell Microbiol 11:11601169

6. Sung MMN, Koonen DPY, Soltys C-LM, Jacobs RL, Febbraio M, Dyck JRB (2011) Increased CD36 expression in middle-aged mice contributes to obesity-related cardiac hypertrophy in the absence of cardiac dysfunction. J Mol Med. doi:10.1007/s00109-010-0720-4

7. Chung SS, Ahn BY, Kim M, Kho JH, Jung HS, Park KS (2010) SUMO modification selectively regulates transcriptional activity of peroxisome-proliferator-activated receptor $\gamma$ in $\mathrm{C} 2 \mathrm{C} 12$ myotubes. Biochem J 433:155-161

8. Rać ME, Safranow K, Poncyljusz W (2007) Molecular basis of human CD36 gene mutations. Mol Med 13:288-296 